\title{
A Case Report: Prolonged Psychosis Secondary to Use of Highly Concentrated Tetra Hydro Cannabinoid (THC) (DABS)
}

\author{
Srinivas Dannaram, Madhuri Pulluri, Christina Regan \\ Banner Thunderbird Medical Center, Department of Psychiatry, College of Medicine-Phoenix, University of Arizona, Glendale, \\ Arizona, USA \\ Email: Srinivas.Dannaram@bannerhealth.com
}

How to cite this paper: Dannaram, S., Pulluri, M. and Regan, C. (2022) A Case Report: Prolonged Psychosis Secondary to Use of Highly Concentrated Tetra Hydro Cannabinoid (THC) (DABS). Open Journal of Psychiatry, 12, 49-52.

https://doi.org/10.4236/ojpsych.2022.121005

Received: October 22, 2021

Accepted: December 28, 2021

Published: December 31, 2021

Copyright $\odot 2022$ by author(s) and Scientific Research Publishing Inc. This work is licensed under the Creative Commons Attribution International License (CC BY 4.0).

http://creativecommons.org/licenses/by/4.0/

\section{(c) (i) Open Access}

\begin{abstract}
The Association of Schizophrenia, psychosis, and Marijuana is decades old. Swedish Conscripts cohort study [1] was one of the earlier studies which reported dose-dependent associations between Marijuana use and schizophrenia. This landmark study was substantiated in other countries through research and systematic reviews [2], which further strengthened the dose-dependent association between Marijuana and Psychosis. The dose defined in these studies [1] was based on the frequency of use that is more times used means more risk of developing schizophrenia and or related psychosis. The concept of using highly concentrated marijuana is relatively new, especially in the light of episodes lasting longer than anticipated with treatment resistance. There is not much data on how it will change the course of psychosis and affect the current diagnostic criteria for Substance-induced psychosis, Schizophreniform Psychosis, and schizophrenia. Based on these challenges, we report a case of Dabs or concentrated Tetra Hydro Cannabinoid (THC)-induced prolonged psychosis with tachycardia and treatment resistance on two separate occasions. Both inpatient admissions were triggered using concentrated Tetra Hydro Cannabinoid (THC).
\end{abstract}

\section{Keywords}

Schizophrenia, Psychosis, Marijuana, THC

\section{Case}

A 23-year-old female with no previous psychiatric illness presented with Paranoid delusions that her mother is playing old movies to pass subliminal messages and sister is changing lights to reflect her mood. She reported that she heard 
planes flying over her house shooting. She also reported hearing derogatory comments made by random people and was feeling stressed and suicidal. The patient has been smoking marijuana since the age of 21, and symptoms started two weeks back since she started to smoke Marijuana Wax pens on average 16 times daily. The patient had a Blood Pressure of 117/85 and a Heart rate of 110 . The urine Toxicology Screen was positive for marijuana. The patient was admitted and was started on Olanzapine, which was gradually increased to a dose of $20 \mathrm{mg}$ with no response; then Fluphenazine $5 \mathrm{mg}$ two times a day was added to which she responded and was discharged on day 18th.

Following discharge, the antipsychotics were discontinued over a few weeks as she was not psychotic anymore. After seven months patient presented with two weeks history of paranoid delusions and auditory hallucinations that Television and Electronic devices are talking to her after using marijuana wax pens for about two weeks. The patient had a Heart rate of 110 - 120, and Urine Toxicology Screen was positive for marijuana. The family was concerned, and after admission, she reported that she might be pregnant. Despite repeating the pregnancy test, she was not convinced. She remained paranoid about other patients, was anxious, and was guilty about using wax pens. The patient was started on Olanzapine $5 \mathrm{mg}$ with no initial change in the severity of symptoms. Patient-reported depressed mood and tearful episodes due to distress associated with psychosis and frustration related to lack of improvement. Lithium was added with no immediate effect after a 20 day stays in the hospital. Prozac was started to address anxiety and depression, and Olanzapine gradually increased to $20 \mathrm{mg}$ with some improvement. The patient continued to have Tachycardia with normal Blood pressure, Electrocardiogram was done twice, and no abnormality was found. Propranolol was added to address autonomic symptoms of anxiety.

The patient was stable by the 38th day in the hospital, educated about the risk of recurrent psychosis with marijuana, and was recommended a Long-acting injectable antipsychotic due to the risk of non-compliance. The patient agreed and attained stability on day 45 .

\section{Discussion}

The legalization of Marijuana with commercialization and technical advancement in purification and production has increased Tetra Hydro Cannabinoid (THC) concentration over time. According to [3] MA ElSohly et al. Tetra Hydro Cannabinoid (THC) content in confiscated samples increased from 3\% in 1980 to $12 \%$ in 2012. Adolescent ED visits due to Marijuana use increased in both males and females by $53.6 \%$ and $42.9 \%$, respectively, from 2005 to 2010 [4]. Highly concentrated Marijuana in the form of Dabs with high Tetra Hydro Cannabinoid (THC) concentrations ranging from $23.7 \%$ to $75.9 \%$ [5] are available legally. One case of Neuro and Cardiotoxicity [6] and two cases of acute psychosis [7] were reported after use of concentrated Tetra Hydro Cannabinoid (THC) extract known as cannabis "wax," "oil," or "dabs." Two acute psychotic 
episodes cases reported by [7] JM Pierre et al. responded to antipsychotics by Hospital Days 12 and 17, respectively.

According to [8] Wyatt et al. Neurotoxicity hypothesis, prolonged or repeated episodes of psychosis leave scars on the brain, possibly via changes in morphology, biochemical alterations, or damage to neuronal connections. This is one of the several mechanisms used to explain the association between the longer duration of untreated psychosis and poorer outcomes [9] [10] [11].

This was backed by a mechanism that involves dopaminergic hyperactivity resulting [12] [13] in prolonged elevation of dopamine leading to a progressive decline in neural volume in regions with concentrated dopamine receptors. Dopaminergic agents and activity also induce neuronal apoptosis [14].

This time-tested evidence proved that the prognosis worsens with significant functional impairment when psychosis is prolonged, frequent, and resistant to antipsychotic treatment. The second case was reported by JM Pierre et al., and our case needed more than one antipsychotic to treat Tetra Hydro Cannabinoid (THC)-induced psychosis. Marijuana is one of the most abused substances with growing popularity in recent decades [15] primarily attributed due to legalization in most states [16]. This, along with an increase in concentration in refined forms, can pose new challenges for the field of medicine with its impact on Central Nervous System, Cardio Vascular System, and Psychiatry, as evident by the cases reported so far.

\section{Conclusion}

The concept of using highly concentrated marijuana is relatively new, especially in the light of episodes lasting longer than anticipated with treatment resistance. There is not much data on how this will change the course of psychosis and affect the current diagnostic criteria for Substance-induced psychosis, Schizophreniform Psychosis, and schizophrenia. Evidence suggests that the prognosis worsens with significant functional impairment when psychosis is prolonged, frequent, and resistant to antipsychotic treatment. Based on these new diagnostic and management challenges, further research in this area is needed to inform new strategies to guide the assessment and management of psychotic disorders triggered by highly concentrated Tetra Hydro Cannabinoid (THC).

\section{Conflicts of Interest}

The authors declare no conflicts of interest regarding the publication of this paper.

\section{References}

[1] Zammit, S., Allebeck, P., Andreasson, S., Lundberg, I. and Lewis, G. (2002) Self Reported Cannabis Use as a Risk Factor for Schizophrenia in Swedish Conscripts of 1969: Historical Cohort Study. British Medical Journal, 325, Article No. 1199. https://doi.org/10.1136/bmj.325.7374.1199

[2] Semple, D.M., McIntosh, A.M. and Lawrie, S.M. (2005) Cannabis as a Risk Factor 
for Psychosis: Systematic Review. Journal of Psychopharmacology, 19,187-194. https://doi.org/10.1177/0269881105049040

[3] ElSohly, M.A., Mehmedic, Z., Foster, S., Gon, C., Chandra, S. and Church, J.C. (2016) Changes in Cannabis Potency over the Last 2 Decades (1995-2014): Analysis of Current Data in the United States. Biological Psychiatry, 79, 613-619. https://doi.org/10.1016/j.biopsych.2016.01.004

[4] Drug Abuse Warning Network (DAWN) (2012) Emergency Department Visits Involving Marijuana among Adolescents Aged 15 to 17: Increase from 2005 to 2010 Varied by Gender.

[5] Raber, J.C., Elzinga, S. and Kaplan, C. (2015) Understanding Dabs: Contamination Concerns of Cannabis Concentrates and Cannabinoid Transfer during the Act of Dabbing. The Journal of Toxicological Sciences, 40, 797-803.

https://doi.org/10.2131/jts.40.797

[6] Rickner, S.S., Cao, D., Kleinschmidt, K. and Fleming, S. (2017) A Little “Dab” Will Do Ya' in: A Case Report of Neuro- and Cardiotoxicity Following Use of Cannabis Concentrates. Clinical Toxicology, 55, 1011-1013. https://doi.org/10.1080/15563650.2017.1334914

[7] Pierre, J.M., Gandal, M. and Son, M. (2016) Cannabis-Induced Psychosis Associated with High Potency "Wax Dabs”. Schizophrenia Research, 172, 211-212.

[8] Wyatt, R.J. (1991) Neuroleptics and the Natural Course of Schizophrenia. Schizophrenia Bulletin, 17, 325-351. https://doi.org/10.1093/schbul/17.2.325

[9] Marshall, M., Lewis, S., Lockwood, A., et al. (2005) Association between Duration of Untreated Psychosis and Outcome in Cohorts of First-Episode Patients: A Systematic Review. Archives of General Psychiatry, 62, 975-983.

https://doi.org/10.1001/archpsyc.62.9.975

[10] Norman, R.M.G., Lewis, S.W. and Marshall, M. (2005) Duration of Untreated Psychosis and Its Relationship to Clinical Outcome. The British Journal of Psychiatry, 48, s19-s23. https://doi.org/10.1192/bjp.187.48.s19

[11] Perkins, D.O., Gu, H., Boteva, K., et al. (2005) Relationship between Duration of Untreated Psychosis and Outcome in First-Episode Schizophrenia: A Critical Review and Meta-Analysis. American Journal of Psychiatry, 162, 1785-1804.

[12] Crespo-Facorro, B., Roiz-Santiáñez, R., Pelayo-Terán, J.M., et al. (2007) Caudate Nucleus Volume and Its Clinical and Cognitive Correlations in First Episode Schizophrenia. Schizophrenia Research, 91, 87-96. https://doi.org/10.1016/j.schres.2006.12.015

[13] Keshavan, M.S., Haas, G.L., Kahn, C.E., et al. (1998) Superior Temporal Gyrus and the Course of Early Schizophrenia: Progressive, Static, or Reversible? Journal of Psychiatric Research, 32, 161-167. https://doi.org/10.1016/S0022-3956(97)00038-1

[14] Simantov, R., Blinder, E., Ratovitski, T., et al. (1996) Dopamine-Induced Apoptosis in Human Neuronal Cells: Inhibition by Nucleic Acids Antisense to the Dopamine Transporter. Neuroscience, 74, 39-50. https://doi.org/10.1016/0306-4522(96)00102-9

[15] Alzghari, S.K., Fung, V., Rickner, S.S., et al. (2017) To Dab or Not to Dab: Rising Concerns Regarding the Toxicity of Cannabis Concentrates. Cureus, 9, e1676. https://doi.org/10.7759/cureus.1676

[16] Hasin, D.S., Sarvet, A.L., Cerdá, M., et al. (2017) US Adult Illicit Cannabis Use, Cannabis Use Disorder, and Medical Marijuana Laws: 1991-1992 to 2012-2013. JAMA Psychiatry, 74, 579-588. https://doi.org/10.1001/jamapsychiatry.2017.0724 